\title{
Les Best Practices, trésor de Troie ou trésor de Barbe Noire?
}

Best Practices : The Treasure of Troy or the Treasure of Black Beard?

¿Los Best Practices : tesoro de Troya o tesoro del pirata Barbarroja?

\section{Colette Dufresne-Tassé}

\section{(2) OpenEdition}

\section{Journals}

Édition électronique

URL : http://journals.openedition.org/iss/414

DOI : $10.4000 /$ iss. 414

ISSN : 2306-4161

\section{Éditeur}

ICOM - International Council of Museums

Édition imprimée

Date de publication : 1 décembre 2015

Pagination : 87-100

ISSN : 2309-1290

Référence électronique

Colette Dufresne-Tassé, «Les Best Practices, trésor de Troie ou trésor de Barbe Noire ? 》, ICOFOM

Study Series [En ligne], 43b | 2015, mis en ligne le 06 février 2018, consulté le 02 mai 2019. URL : http:// journals.openedition.org/iss/414; DOI : 10.4000/iss.414 


\title{
Les Best Practices, trésor de Troie ou trésor de Barbe Noire?
}

\author{
Colette Dufresne-Tassé
}

\author{
Université de Montréal - Canada
}

Pour des raisons demeurées obscures, le Directeur du Conseil international des musées (ICOM) formule en 2010 le souhait que les Comités internationaux ${ }^{32}$ se dotent de best practices. L'un de ces comités, le Comité pour l'éducation et l'action culturelle (CECA), se montre intéressé, mais avant de s'engager, demande qu'on lui fournisse une définition. Les best practices étant un phénomène bien connu, trouver cette définition semble de prime abord un exercice banal. En fait, il s'avèrera assez lourd, et son résultat, pour le moins étonnant et problématique. En effet, il n'aboutira pas à l'identification d'une catégorie précise de pratiques, les meilleures, mais à une constellation de types de pratiques, dont la valeur varie grandement et dont l'exercice peut engendrer des problèmes non négligeables, si bien qu'il y a lieu de s'interroger sur l'intérêt pour les Comités internationaux à forte orientation professionnelle de recueillir des best practices et d'en diffuser. En effet, s'il ne s'agit pas vraiment de pratiques exemplaires que l'on peut employer comme telles ou dont on peut s'inspirer sans crainte. Quelle est leur utilité ?

Le présent article fait état de la recherche bibliographique réalisée sur les best practices, de ses résultats et du questionnement que ceux-ci soulèvent. II est par ailleurs un complément intentionnel à l'article de Marie-Clarté O'Neill intitulé : « Développement d'un outil international de pilotage des programmes éducatifs et culturels: L'instrument d'analyse « Best Practice » ICOM/CECA », publié dans cet ouvrage.

\section{À la recherche d'une définition, une démarche qui s'allonge}

La stratégie la plus simple et la plus rentable consistait à passer en revue les publications issues du milieu muséal, car il aurait dû en émerger une définition à peu près appropriée et ne nécessitant que de minimes retouches. Déception. Les publications étaient peu nombreuses et leur contenu, qui semblait emprunter à d'autres domaines, offrait peu d'éléments susceptibles de contribuer à la rédaction d'une définition. Une recherche plus vaste s'imposait.

L'étude réalisée visera tous les domaines ayant publié un texte sur les best practices au premier juillet 2011 sous forme électronique ou papier et dans les trois langues officielles de l'ICOM, soit l'anglais, l'espagnol et le français. Le nombre de sites web identifiés sera de plusieurs milliers. Quant à celui des publications-papier, il sera plutôt de l'ordre des centaines. Mais le nombre de textes offrant une définition ou de l'information basale, comme la façon d'étudier une pratique ou d'en déterminer la qualité, se limitera à une soixantaine

\footnotetext{
${ }^{32}$ Chacun des 31 Comités internationaux de l'ICOM est affecté à un aspect du travail muséal, au fonctionnement d'un type de musée ou aux problèmes particuliers d'un type de collection.
} 
dans le cas des sites web et à une douzaine dans celui des publications-papier. Ils livreront l'origine des best practices et leur évolution, leurs caractéristiques principales, des façons de les sélectionner, un ensemble de sous-catégories de pratiques, ainsi que les avantages et les problèmes que pose leur utilisation. Le texte qui suit aborde chacun de ces sujets et se termine par une réflexion sur l'intérêt pour les Comités internationaux de l'ICOM d'orientation professionnelle marquée de recueillir et de diffuser des best practices.

\section{Remarques}

1. Comme à l'accoutumée, toutes les publications utilisées sont listées à la fin de cet article sous la rubrique « références ". Toutefois, elles ne sont pas nommément citées dans le corps de l'article parce qu'elles sont la plupart du temps fort nombreuses à venir en appui à un aspect traité. De plus, elles se répètent constamment, de sorte qu'elles risquaient de couper le fil de la lecture sans apporter en contrepartie une information substantielle.

2. L'équivalent espagnol de «best practices" est "buenas practicas », alors que l'équivalent français est «pratiques exemplaires ». C'est cette dernière expression qui sera dorénavant employée.

\section{Origine et évolution des pratiques exemplaires}

Les pratiques exemplaires semblent originaires du domaine de la gestion et atteindre un haut niveau de reconnaissance dans les années 1990. C'est l'époque où un grand nombre de domaines comme le droit, l'ingénierie, la psychologie, le service social, le nursing, la médecine ou l'environnement les perçoivent comme importantes et en diffusent un grand nombre. Mais dans plusieurs secteurs, on devient sceptique quand on découvre qu'une pratique, même de très haute qualité, peut poser des problèmes de transfert du site où elle a été produite à un autre. II s'ensuit une période de relatif désenchantement qui persiste encore, mais qui voit tout de même de nouveaux domaines, comme la gouvernance locale, l'intervention sociale ou l'agriculture, rejoindre les rangs des diffuseurs de pratiques exemplaires. Par ailleurs, l'un des modes de sélection de ces pratiques, le benchmarking, connaît actuellement une vogue exceptionnelle comme outil de gestion des entreprises et même des institutions.

\section{Caractéristiques communes}

Une pratique exemplaire est soit une façon de procéder, soit une production. Une façon de procéder peut prendre la forme d'une technique, d'une procédure, d'une méthode ou même d'une stratégie. En muséologie, ce pourrait être : une technique de restauration, une manière de préparer un scénario d'exposition, une stratégie de développement de collection ou de public. Par ailleurs, on peut considérer comme une production un produit tangible ou une action, en d'autres termes une intervention susceptible de prendre plusieurs formes, comme le feraient en muséologie une visite guidée, un programme culturel, une exposition ou un catalogue.

Pour obtenir l'appellation exemplaire, une pratique - façon de procéder ou production - doit posséder les quatre caractéristiques suivantes :

1) Être efficace : ses effets doivent être évidents, stables et persistants. Ils ne peuvent bien entendu l'être que si la 
pratique est adaptée à la situation pour laquelle elle a été conçue;

2) Être transférable à d'autres situations: pour que ce soit possible, il faut que les contextes dans lesquels interviennent les professionnels aient un haut niveau de similitude ;

3) Être innovante: plus précisément, être inédite. En outre, cette nouveauté doit présenter une certaine simplicité et une certaine harmonie, c'est-à-dire de l'élégance ;

4) Être évolutive : pour demeurer exemplaire, une pratique doit pouvoir évoluer en fonction des trois facteurs suivants: l'accroissement des connaissances ou l'apparition d'innovations techniques, l'évolution de l'institution où la pratique a été conçue et les besoins ou demandes des usagers.

\section{Modes de sélection}

Avant d'accéder au rang de pratique exemplaire, toute pratique doit être examinée, et on ne l'étudie qu'à partir d'une description formelle. Dans certains domaines, on n'impose qu'une seule contrainte: la description doit être détaillée et exhaustive. Dans d'autres, par contre, on exige que le compte rendu obéisse à des consignes précises et même s'insère dans un formulaire ou dans une trousse de description ou template. Demandes, formulaires ou trousses ont toutefois les mêmes buts: obtenir des descriptions similaires fournissant suffisamment d'informations pour que l'on puisse s'assurer de leur valeur et les comparer.

Mais la façon dont on procède pour apprécier l'information que l'on possède sur une pratique varie beaucoup. Elle diffère selon ceux qui la jugent, les aspects qu'ils en considèrent et les moyens qu'ils utilisent pour l'évaluer.

Qui juge? Une autorité désignée par le domaine ; la plupart du temps, c'est un jury. Toutefois celui-ci peut être constitué soit d'experts, soit de pairs.

Quels aspects d'une pratique examine-t-on? Le jury est libre de considérer des aspects généraux, des aspects majeurs ou, au contraire, une série d'aspects précis habituellement indiqués dans une grille d'analyse.

Quels moyens utilise-t-on pour se prononcer sur les aspects à évaluer? Pour prendre sa décision, un jury peut utiliser trois sortes de moyens très différents :

1) Le jugement de ses membres, qui s'inspire habituellement de valeurs ou de standards, c'est-à-dire de principes ou de règles qui, s'ils ne font pas consensus, sont au moins généralement acceptés dans le domaine ;

2) La comparaison rigoureuse de la façon de procéder ou de la production considérée avec d'autres façons de procéder ou d'autres productions estimées exemplaires (procédé appelé en anglais benchmarking ) ;

3) Des critères d'origines variées:

a. Déterminés par des spécialistes ; ils sont habituellement dérivés d'entrevues avec ceux-ci ;

b. Issus des résultats de la recherche connus à travers l'analyse des publications scientifiques ;

c. Issus à la fois des résultats de la recherche, auxquels peuvent s'ajouter l'évaluation formelle des conséquences 
de la pratique étudiée et/ou l'avis des praticiens qui s'en servent. ${ }^{33}$

Commentaire: Comme un jury semble n'utiliser qu'un seul moyen d'évaluation et que les moyens précédents comportent des exigences différentes, on peut supposer que les pratiques sélectionnées comme exemplaires possèdent les quatre caractéristiques décrites plus haut à des degrés différents.

\section{Types de pratiques exemplaires}

Dans les publications consultées, on peut distinguer trois types, de sorte qu'une pratique exemplaire peut être la meilleure, la plus appropriée ou la plus généralement acceptée.

La pratique (façon de procéder ou production) est la meilleure. Cela signifie qu'elle est la plus prudente, la plus sûre, et qu'elle donne les résultats les plus recherchés. La sélection de ce type de pratique suppose la comparaison d'un nombre élevé de pratiques, et celle qui ressort non seulement possède les quatre caractéristiques communes à toute pratique exemplaire - efficacité, transférabilité, nouveauté, flexibilité - de manière remarquable, mais à un degré qui surpasse toutes les autres.

La pratique (façon de procéder ou production) est la plus appropriée. En d'autres mots, vu les situations considérées, c'est celle qui convient le mieux. Dans ce cas-ci comme dans le cas précédent, il y a évaluation, mais les exigences sont différentes. On n'exige plus d'une pratique qu'elle possède les quatre caractéristiques à un niveau exceptionnel. Elle doit seulement les détenir à un très haut niveau, car ce qui compte surtout c'est que, comme pratique, elle convienne vraiment - en fait mieux que les autres - aux situations considérées.

La pratique (façon de procéder ou production) est la plus généralement acceptée, de sorte que c'est celle que l'on utilise habituellement.

Commentaire concernant les pratiques généralement acceptées: Selon que le domaine est plus ou moins ancien, évaluation et comparaison, sans que cela soit clair dans les publications consultées, semblent se réaliser différemment, et la signification de l'appellation généralement acceptée s'en trouve modifiée.

a. Le domaine est ancien. Évaluation et confrontation des pratiques sont alors implicites, car elles ont eu lieu il y a si longtemps qu'on les a quasiment oubliées. C'est ce qui se passe dans le cas de certains procédés de fabrication, tel celui de l'acide sulfurique, qui ont acquis un statut presque paradigmatique. La pratique considérée est alors devenue, à quelques détails près, une pratique généralisée ;

b. Le domaine est plutôt récent. Alors évaluation et comparaison ne sont pas systématiques ; elles se réalisent à mesure que les professionnels mettent au point des façons de faire ou des productions qui leur semblent plus satisfaisantes. II en résulte une espèce de sélection dans le temps des pratiques, de sorte qu'à un moment précis l'une d'elles est la plus communément employée ;

\footnotetext{
${ }^{33}$ L'avis des praticiens est utilisé surtout dans les domaines de la médecine et du nursing.
} 
c. Le domaine est émergent. Dans ce cas, évaluation et comparaison sont minimales, car les pratiques sont encore en cours de structuration et peu nombreuses. Si l'une d'entre elles donne des résultats considérés comme intéressants, ce jugement est habituellement temporaire, car avec l'évolution du domaine on développera à son endroit et vis-à-vis de ses résultats des exigences plus précises et plus fortes.

\section{Commentaires concernant les trois types de pratiques exemplaires}

a. Nulle part dans les publications consultées on ne précise pourquoi, dans un domaine, on parle de meilleure pratique, alors que dans d'autres il est question de pratique appropriée ou généralement acceptée. Je fais l'hypothèse que c'est à cause de l'état dans lequel se trouve le domaine. Pour qu'il puisse produire des pratiques dites les meilleures, ou les plus généralement acceptées, il doit avoir atteint une certaine maturité, c'est-à-dire créé les conditions nécessaires pour que les façons de faire y aient atteint un haut niveau de perfectionnement, en d'autres mots, que des pratiques plus ou moins adéquates se soient améliorées lors d'emplois successifs, qu'elles aient acquis une forme à peu près stable dont on saisit le potentiel, de sorte que peuvent émerger certaines qui sont à la fois hautement utiles, innovantes et flexibles. En outre, le domaine doit avoir stabilisé les conditions de travail de ses professionnels au point où elles présentent une similitude suffisante au transfert d'une pratique d'un endroit à un autre. Dans les domaines jeunes ou en émergence, les pratiques sont encore peu perfectionnées, sinon en pleine formation, de sorte que l'apparition de pratiques considérées comme les meilleures ou les plus généralement acceptées y est pratiquement impossible. Par contre, dans les domaines anciens, on devrait pouvoir observer des pratiques considérées comme les plus appropriées à certaines situations.

b. Quel que soit le type de pratique exemplaire considéré, la variation des modes d'évaluation notée plus haut entraîne des différences appréciables du niveau d'utilité, de nouveauté, de transférabilité et de capacité évolutive des pratiques sélectionnées. La fluctuation des modes d'évaluation influe aussi probablement sur d'autres caractéristiques des pratiques exemplaires, telles leur rigueur, leur adhésion à des connaissances scientifiques de pointe, leur respect de valeurs ou de chartes, comme celle des libertés et des droits fondamentaux de la personne. Ces caractéristiques ne sont pas nommément mentionnées dans les sources dont les références apparaissent à la fin de ce texte. Je les ai déduites de leur lecture et, pour cette raison, je les appelle périphériques.

Avantages d'une utilisation intensive des pratiques exemplaires Les partisans des pratiques exemplaires, surtout ceux qui promeuvent "les meilleures» - ils étaient plus nombreux dans le passé que maintenant -, considèrent qu'un domaine qui en développe s'enrichit. II s'améliorerait intellectuellement, éthiquement et organisationnellement.

Les gains intellectuels sembleraient:

- L'accumulation d'informations sur des techniques et des méthodes ;

- Des interventions ou des programmes efficaces ; 
- L'explication de leur efficacité ;

- L'identification des situations pour lesquelles une pratique est pertinente.

Les gains éthiques seraient doubles. La multiplication des pratiques exemplaires permettrait en effet :

- D'en tirer des principes ou des directives générales pour le domaine ;

- De contribuer à la transparence des interventions des agences ou des organismes qui agissent au nom de l'État.

Quant aux gains organisationnels, les principaux seraient les suivants :

- Une amélioration des performances de l'entreprise ou de l'institution se traduisant par l'offre de meilleurs produits ou de meilleurs services à la population;

- Une économie de temps et une diminution de coûts ;

- Une amélioration de l'image de l'institution et un accroissement de sa visibilité.

\section{Problèmes}

Les invitations à la prudence, peu nombreuses au moment de la diffusion des premières pratiques exemplaires, se sont ensuite multipliées. Aujourd'hui, elles portent surtout sur les points suivants :

- Les risques inhérents au transfert de pratiques issues d'institutions dont la culture d'entreprise est tout à fait particulière ;

- Les exigences techniques contraignantes de certaines pratiques, en particulier celles des pratiques qui requièrent des prérequis importants.

\section{Synthèse}

\section{Les pratiques exemplaires, une constellation protéiforme}

À l'issue de la présentation que l'on vient de lire, les pratiques exemplaires apparaissent comme un genre déterminé par quatre caractéristiques, quatre qualités centrales: l'efficacité, la transférabilité, la nouveauté et la capacité d'évoluer. Ce genre constitue en réalité une constellation tout à fait protéiforme de pratiques que l'on peut classer dans de multiples catégories déterminées par le jeu des facteurs de différenciation suivants :

- La nature même des activités professionnelles considérées: façon de faire versus production ;

- Leur type: meilleure pratique, pratique la plus appropriée, pratique la plus généralement acceptée ;

- Les juges - experts ou pairs - le mode d'évaluation qu'ils privilégient et l'importance relative qu'ils accordent à chacune des caractéristiques centrales (utilité, nouveauté, transférabilité, flexibilité) et aux caractéristiques périphériques (rigueur, actualité scientifique, respect de valeurs ou de chartes, etc.).

En somme, on se trouve devant une nébuleuse de pratiques qui diffèrent de multiples façons, y compris dans leur niveau d'excellence. II n'est donc probablement pas exagéré de dire qu'il y a pratique exemplaire et pratique exemplaire, et de s'interroger sur la signification du terme exemplaire dans un tel contexte. 


\section{Une réflexion obligée}

Comme on vient de le constater, l'étude bibliographique dont on vient de lire les résultats suscite une série d'interrogations et incite à la proposition d'alternatives à la collecte et à la diffusion de pratiques exemplaires.

\section{Des interrogations}

Question 1: Comment un Comité international de I'ICOM doit-il considérer des pratiques exemplaires dont la valeur même peut grandement fluctuer?

Question 2: Que recherche un comité à travers la diffusion de ces pratiques ? Promouvoir l'excellence en l'honorant et en la diffusant largement? Faire reconnaître le dynamisme de son secteur et éventuellement élargir son champ d'action ? Veut-il aussi, ou plutôt, utiliser des pratiques exemplaires pour améliorer ses méthodes et son offre au public ? Si c'est le cas, prend-il le bon moyen ?

Question 3: Le comité qui encadre un secteur du travail muséal considère-t-il que celui-ci possède la maturité suffisante pour produire des pratiques à la fois hautement efficaces, nouvelles et susceptibles d'évoluer?

Question 4: Le secteur possède-t-il une similarité de conditions de travail suffisante pour que le transfert soit non seulement possible, mais fructueux? Vu que les Comités internationaux de l'ICOM ont des membres dans toutes les régions du monde, vu également que les conditions de travail d'un professionnel dépendent à la fois des besoins ou des attentes auxquelles il doit répondre et des ressources de son institution, on doit se demander si ces paramètres sont semblables d'une région du monde à l'autre. S'ils ne le sont pas, quelle est l'utilité de pratiques diffusées internationalement?

Question 5 : La publication de pratiques exemplaires ne risque-t-elle pas d'encourager les professionnels à les copier plutôt qu'à élaborer leurs propres productions? Dans le cas des expositions et des programmes culturels par exemple, cette reproduction serait contreproductive, car elle engendrerait leur uniformisation au moment même où l'on tente de les diversifier, pour proposer au visiteur fréquent, qui se déplace de plus en plus, de la nouveauté où qu'il aille.

Question 6: Un Comité international qui diffuserait beaucoup de pratiques exemplaires ne risquerait-il pas d'enfermer son secteur dans un empirisme facile, ses professionnels se contentant de reprendre « ce qui marche » sans trop savoir pourquoi ?

Question 7 : Autre crainte, mais cette fois au sujet du benchmarking. Ce moyen de sélection, on l'a vu, consiste à comparer une pratique à d'autres considérées comme des standards d'excellence. Son emploi dans plusieurs domaines, dont celui de la gérance des grands services ou des institutions de l'État, a eu des conséquences désastreuses. ${ }^{34}$ On s'est en effet aperçu que services et institutions se sont mis à rechercher le «bien total », c'est-à-dire à tenter d'atteindre et si possible de dépasser ce qui se fait de mieux au monde, plutôt que de réaliser le mieux possible ce qu'ils doivent faire pour la population qu'ils desservent. Quant aux professionnels engagés dans cette course à la performance, la plupart ont perdu le

${ }^{34}$ Voir par exemple : Bruno, 2008, 2010 ; Bruno \& Didier, 2013. 
sens de leur mission et souffert d'épuisement récurrent. Le milieu muséal ne pâtirait-il pas lui aussi de cette compétition malsaine s'il se mettait à développer et à diffuser systématiquement des pratiques exemplaires?

\section{Une alternative à la diffusion de pratiques exemplaires}

$\mathrm{Si}$ les Comités internationaux de l'ICOM d'orientation fortement professionnelle possèdent la maturité nécessaire au développement de pratiques exemplaires, leur diffusion constitue probablement un bon moyen de faire connaître leur dynamisme, car, malgré ce que l'on en sait, ces pratiques conservent une aura positive. Toutefois, cet avantage n'exclut pas les risques qui viennent d'être évoqués.

A contrario, la diffusion des trois instruments suivants : un catalogue des aspects à considérer au moment où l'on conçoit, réalise et évalue une production muséale, des recommandations sur le type d'information à recueillir à chacun de ces moments et la rédaction d'un journal de terrain sont susceptibles de conduire à une amélioration constante des façons de procéder d'un professionnel ou d'une équipe muséale. En outre, l'emploi de cet ensemble d'instruments devrait favoriser leur créativité.

Un catalogue des aspects à considérer constitue un guide qui accompagne la conception, la réalisation et l'évaluation d'une production muséale, comme un programme culturel, une exposition ou un site web (voir un exemple de catalogue visant un programme dans l'article de Marie-Clarté O'Neill publié dans le même ouvrage). Ce guide fournit aussi, en fin de conception, un aide-mémoire qui permet de vérifier si tous les aspects qui devaient être pris en compte l'ont été. II évite ainsi les défauts de facture les plus courants et garantit à la production une qualité minimale. ${ }^{35}$

Quant aux recommandations sur l'information nécessaire à la concrétisation de chaque aspect d'une production, elles contribuent grandement à en assurer la pertinence, la richesse et la valeur scientifique, en d'autres termes, la valeur muséale. Par exemple, la connaissance des caractéristiques socio-économico-culturelles du milieu dans lequel le musée est intégré concourt grandement à la pertinence d'un programme destiné au public de ce milieu, celle de l'information scientifique sur le sujet qu'il traite garantit l'exactitude et la richesse du programme, alors que celle de l'éventail des outils de médiation disponibles permet la meilleure adaptation possible des moyens sélectionnés au contenu à diffuser, aux caractéristiques des bénéficiaires et aux conditions dans lesquelles le programme est offert.

Aux bénéfices précédents, s'en ajoutent d'autres, plus généraux, qui aident le musée à remplir ses obligations à titre d'institution ${ }^{36}$ intégrée dans une société. Les principaux sont les suivants :

a. Guider l'action du musée et l'ajuster aux attentes de la société tout en influençant le développement de cette dernière selon une orientation précise ;

\footnotetext{
${ }^{35}$ Éventuellement, ce catalogue pourrait aussi servir à évaluer des pratiques candidates au titre de pratique exemplaire.

${ }^{36}$ En s'inspirant des textes de Calvez (2006), de Laforgue (2009) et de Nantel (2010), on peut considérer un musée comme une institution, c'est-à-dire comme un ensemble de ressources matérielles et humaines rassemblées par une société ou par des individus au nom de cette dernière, qui doit assurer sa persistance tant qu'elle est utile, de façon à rendre des services, mais aussi à faciliter et orienter le développement de la société.
} 
b. Assurer à chaque moment une offre de la plus haute qualité possible, basée sur les résultats de la recherche et les données de l'expérience ;

c. Comprendre comment et pourquoi le musée parvient à faire cette offre, mais aussi les lacunes que celle-ci comporte malgré tout ;

d. Remédier à ces lacunes et développer d'autres offres ;

e. Etre bien géré et se maintenir comme institution efficace et comme ressource importante de la société.

Le journal de terrain est l'endroit où l'on note le déroulement de chacune des interventions qui contribuent à la conception, à la réalisation et à l'évaluation d'une production muséale. Mais c'est aussi le lieu où l'on consigne les modifications que l'on a dû apporter au projet initial et leurs raisons, les incidents qui ont changé son déroulement et provoqué des résultats inattendus. En somme, le journal de terrain rassemble des informations qui contribuent grandement à saisir l'évolution d'une production et à en expliquer les résultats.

Par ailleurs, la rédaction de notes est aussi l'occasion de réfléchir sur les problèmes que l'on a observés, et d'imaginer, par exemple des transformations qui simplifieraient ou amélioreraient une façon de procéder, de saisir le potentiel non exploité d'un instrument ou d'un type de situation, ce qui relance automatiquement le questionnement. Le journal de terrain est ainsi une source d'inspiration, de dynamisme et de renouvellement des façons de faire d'un professionnel ou d'une équipe. Facteur de compréhension d'une production et de ses résultats, mais aussi facteur de créativité, il apparaît donc comme un complément obligé des deux autres instruments proposés.

En réalité, lorsqu'ils utilisent conjointement les trois instruments de façon constante, un professionnel et son équipe se trouvent à réaliser de la recherche appliquée. Ce type de recherche soutient, on vient de le voir, l'évolution continue de leurs façons de procéder, tout en assurant à la fois leur rigueur et leur qualité. Par ailleurs, cette recherche appliquée est constamment liée à la recherche fondamentale. En effet, les informations recueillies durant la conception des productions muséales sont tirées en bonne partie de la recherche fondamentale qui, en retour, s'enrichit des données obtenues sur les effets ou les conséquences des productions réalisées.

Si des institutions situées dans chacune des régions du monde utilisaient systématiquement les trois instruments proposés durant quelques années, qu'obtiendrait-on ? On possèderait, pour chaque région, un ensemble de façons d'exécuter la conception, la réalisation et l'évaluation de productions muséales à la fois rigoureuses, originales et efficaces parce qu'adaptées aux caractéristiques des institutions, aux publics auxquels celles-ci s'adressent et aux contextes dans lesquels elles interviennent. Une enquête ou même un colloque permettrait alors d'identifier des éléments communs qui deviendraient une sorte de modèle, assorti de multiples variantes dont l'éventail pourrait s'élargir.

\section{Les pratiques exemplaires, trésor de Barbe Noire ?}

Dans la mesure où les secteurs de l'ICOM à forte orientation professionnelle possèdent suffisamment de maturité pour produire des pratiques exemplaires, leur diffusion peut être une source de prestige. Toutefois persiste pour ces secteurs le risque d'une 
compétition malsaine, d'un épuisement récurrent de leurs professionnels, d'une diminution de leur créativité et de leur adhésion à un empirisme potentiellement sclérosant. En ce qui concerne les secteurs jeunes ou en émergence, on l'a vu, les pratiques exemplaires ont pour eux peu de sens. Par contre, les trois instruments d'accompagnement de la conception, de la réalisation et de l'évaluation d'une production muséale sont adaptés à tous les secteurs, quel que soit leur niveau d'évolution. En effet, en soutenant de la recherche appliquée, ils peuvent être à l'origine d'un processus d'évolution et d'amélioration de leurs façons de faire, quels que soient les acquis dont ils jouissent déjà. II est donc permis de se demander si, comparées aux trois instruments proposés, les pratiques exemplaires ne perdent pas leur aura de trésor fabuleux et ne ressemblent pas un peu aux quelques sacs de sucre, à la farine avariée et aux explosifs pourris que contenait la cache du célèbre pirate américain que fut Barbe Noire?

Note: Certains des aspects du présent texte ont été publiés dans l'article suivant: Dufresne-Tassé, C. (2012). État de la question sur les pratiques exemplaires (best practices). CADMO Giornale Italiano di Pedagogia Sperimentale, Anno XX, 1, p. 5-22.

\section{Références sur les « best practices »}

\section{Publications-papier}

American Association of Museums (2008). National Standards \& Best Practices for U.S. Museums. Washington, DC: The American Association of Museums.

American Association of Museums (2011). "What Does "Benchmarking" Mean, and Why Should We Bother with Benchmarking Research? " Museum, May/June, 33.

Griffin, A. (1997). " PDMA Research on New Product Development Practices: Updating Trends and Benchmarking Best Practices. "The Journal of Product Innovation Management, vol. 14, 429-458.

Médiation culturelle association (2008). Charte déontologique de la médiation culturelle 1. Introduction et principes de la médiation culturelle (2004-2007). Lyon, FR: Médiation culturelle Association.

Reusner, E.M. (2004). "Best Practices in Audience Research and Evaluation. Case Studies of Australian and New Zealand Museums. ॥ Visitor Studies Today, vol. VII, no 2, 17-26.

Silow-Carol, S., Alteras, T., \& Stepnick, L. (2006). Patient-Centered Care for Underserved Populations: Definition and Best Practices. Report prepared for the W.K. Kellogg Foundation. Washington, DC: Economic and Social Research Institute.

Smith Nash, S. (2005). « Learning Objects, Learning Object Repositories and Learning Theory: Preliminary Best Practices for Online Courses. " Interdisciplinary Journal of Knowledge and Learning Objects, vol. 1, 218-228.

Soren, B. J. (2005). « Best Practices in Creating Quality Online Experiences for Museum Users. " Museum Management and Curatorship, vol. 20, no. 2, 131-148.

Standing Professional Committee on Education of the American Association of Museums (2005). Excellence in Practice: Museum Education Principles and Standards. Washington, DC: American Association of Museums.

Szulanski, G. (1996). «Exploring Internal Stickiness: Impediments to the Transfer of Best Practices within the Firm. " Strategic Management Journal, vol .17, Special issue: Knowledge and the Firm, 27-43.

The State Historical Society of lowa (2004). Fundamental Best Practices that Every Museum Can Implement Regardless of Financial or Staff Limitations. Des Moines, OA: State Historical Museum. 
Turner, D. M., Haley, H., \& Hallencreutz, J. (2009). «Towards a Global Definition of Best Practice in Change Management. " The International Journal of Knowledge, Culture \& Change Management, vol. 9, no. 8, 185-191.

Zemelman, S., Daniels, H., \& Hyde, A. (2005). Best Practice. Today's Standards for Teaching \& Learning in America's Schools. Portsmouth, $\mathrm{NH}$ : Heineman.

Sites provenant du milieu muséal (consultation des sites le 15 juillet 2011)

Alberta Association for Supported Employment (AASE) Best Practices. http://aase.ca/pdf/best_practices.pdf

American Association of Museums. http://www.speakupformuseums.org/docs/legpriorities/BREIF\%20Eth ics $\% 20$ Best $\% 20$ Practices.pdf

British Columbia Museums Association. http://www.museumsassn.bc.ca/Images/Best $\% 20$ Practices $\% 20 \mathrm{Mod}$ ules $\% 202 /$ Revenue $\% 20$ Generation\%20FINAL.pdf

Conserving Digital Art for the Ages. http://www.google.ca/search?client=safari\&rls=en\&q=best+practice+ museum+definition\&ie=UTF-8\&oe=UTF8\&redir esc=\&ei=W3Y5Tv3iLcb00gG53u35Aw

Government of Australia Western Australian Museum. http://www.museum.wa.gov.au/research/developmentservice/\#introduction

International Zoo Educators Association. http://www.izea.net/education/guidelines_ed_museums.htm

Museum Association Museum Practice. http://www.museumsassociation.org/museum-practice

Museum-Ed. http://www.museumed.org/index.php?option=com_content\&view=article\&id=102:bestpractices-in-museum-exhibition-writing-2010\&catid=37: currentpractice-interpretation\&ltemid $=86$

Museum Libraries and Archives. http://www.mla.gov.uk/what/raising_standards/best_practice

My Canadian Museum Reference for Technologies. http://www.pro.rcipchin.gc.ca/contenu_numeriquedigital_content/preservation_numerique-digital_preservation/guidesbestpractice-eng.jsp

UNESCO Office in Venice. http://www.unesco.org/new/en/venice/about-thisoffice/single-

view/news/best_practices_in_museum_management_dealing_with difficult_heritage educating_on history_in_south_east_europe_berli n_germany_27_28_january_2011/

University of Victoria Best Practices in Museum Site Design. http://www.uvcs.uvic.ca/crmp/museumwebsites/concept_6_introducti on.aspx

Webographie sur les Best practices (consultés entre le 2 et le 15 juillet 2011)

Active Citizenship Network. Page consultée au http://www.activecitizenship.net/files/european_day/2011/european_ conference/best_practices_final_report.pdf

Answers.com Best Practices Benchmarking and Consulting, LLC. Page consultée au http://www.answers.com/topic/best-practice

$\begin{array}{cc}\text { ASME. } & \text { Page } \\ & \text { http://files.asme.org/asmeorg/Governance/Institutes/7990 }\end{array}$

Best-Practice.com. Page consultée au http://www.best-practice.com/

Best Practices Florida Education. Page consultée au http://education.bestpracticesfl.com/definition.html

Best Practices Guides. Page consultée au http://www.bestpracticeguides.org.uk/health_definewelfare.aspx

Best Practices in Health Promotion: The Interactive Domain Model (IDM). Page consultée au http://www.bestpracticeshealthpromotion.com/id12.html

Bitpipe.com. The TechTarget Library of White Papers, Product Literature, Webcasts and Case Studies. Page consultée au http://www.bitpipe.com/tlist/Best-Practices.html 
Business Dictionary.com. Page consultée au http://www.businessdictionary.com/definition/best-practice.html

Cambridge Dictionaries Online. Page consultée http://dictionary.cambridge.org/dictionary/british/best-practice

CFA Institute, Investopedia. Page consultée http://www.investopedia.com/terms/b/best_practices.asp

Collins English Dictionary C Dictionary.com. Page consultée au http://dictionary.reference.com/browse/best+practice

Commerce Dictionary.com. Page consultée au http://www.commercedictionary.com/definition/best-practice.html

Community Mental Health Oakland County Authority. Page consultée au http://www.occmha.org/index.php?option=com_content\&view=article \&id $=117$

Derek Stockley. Page consultée au http://derekstockley.com.au/newsletters05/042-best-practice-consulting.html

Education.com. Page consultée au http://www.education.com/definition/bestpractice/

eHow. Page consultée au http://www.ehow.com/about_5048440_definitionevidence-based-practice.html

ELECTRI Knowledge Network. Page consultée au $\mathrm{http}: / /$ www.electri.org/ekn/ecf home.aspx

Health Canada. Best Practices - Treatment and Rehabilitation for Seniors with Substance Use Problems. Page consultée au http://www.hcsc.gc.ca/hc-ps/pubs/adp-apd/treat_senior-trait_ainee/backgroundcontexte-eng.php

Human Resources iQ. Page consultée au http://www.humanresourcesiq.com/glossary/best-practices/

HyperMedia Corporation. Page consultée au http://www.walden3d.com/best_practices/bp_organization_perspecti ve.htm

ITSMF Australia. Page consultée au http://www.itsmf.org.au/bestpractice/about-best-practice/

K4Health Knowledge for Health. Page consultée au http://info.k4health.org/practices.shtml

LAWperationnel. Page consultée au http://www.lawperationnel.com/Dictionnairelng/Best\%20practice.htm

Linguee.fr Le web est un dictionnaire. Page consultée au http://www.linguee.fr/anglaisfrancais/traduction/definition+of+best+practice.html

Longman Dictionary of Contemporary English. Page consultée au http://www.Idoceonline.com/dictionary/best-practice

MACMILLAN Dictionary. Page consultée au http://www.macmillandictionary.com/dictionary/british/best-practice

Mediamind Eyeblaster Whitepapers. Page consultée au http://www.mediamind.com/Data/Uploads/whitepapers/Eyeblaster White_Paper\%20-\%20Video_Best_Practices\%20_5.pdf

National Council of State Boards of Nursing. Page consultée au https://www.ncsbn.org/Evidence_based_HC_Nsg_Regulation_updat ed_5_07_with_name.pdf

Oxford Dictionaries. Page consultée au http://oxforddictionaries.com/definition/best+practice?region=us

PLM Interest Group. Page consultée au http://www.plmig.com/welcome/stdplmbestpractice.shtml

Project Kikstart. Page consultée au http://www.projectkickstart.com/downloads/tips10-projectmanagement-best-practices.

PsychCentral. Page consultée au http://www.reliabilityweb.com/art04/best_practices_pm.

Registered Nurses' Association of Ontario. Page consultée au http://www.rnao.org/pda/risk/page13.html

Reliability web.com. Page consultée au http://www.reliabilityweb.com/art04/best_practices_pm.htm

Quality Assurance Solutions. Page consultée au http://www.qualityassurance-solutions.com/index.html

QFINANCE The ultimate financial resource. Page consultée au http://www.qfinance.com/dictionary/best-practice 
SearchSoftwareQuality.com Page consultée au http://searchsoftwarequality.techtarget.com/definition/best-practice

TCGen. Page consultée au http://www.tcgen.com/414/social-multimedia--abest-practice-for-product-definition/

Page consultée au http://www.technopedia.com/definition/14269/best-practice

The Free Dictionary. Page consultée au http://encyclopedia2.thefreedictionary.com/best+practices

Treasury Board of Canada Secretariat (1999). Review of Canadian Best Practices in Risk Management. Page consultée au http://www.tbssct.gc.ca/pubs_pol/dcgpubs/riskmanagement/rm-rcbp01-eng.asp

United Nations Educational, Scientific and Cultural Organization. Page consultée au http://www.unesco.org/most/bpindi.htm

USLegal Uslegal.com Page au http://definitions.uslegal.com/b/best-practices/

Visitask.com Project management training and resources. Page consultée au http://www.visitask.com/best-practice-g.asp

Webopedia. Page consultée au http://www.webopedia.com/TERM/B/best_practices.html

Wikipedia The Free Encyclopedia. Page consultée au http://en.wikipedia.org/wiki/Best_practice

Your Dictionary Business. Page consultée au http://business.yourdictionary.com/best-practice

Références sur le benchmarking

Bruno, I. (2008). "La recherche scientifique au crible du benchmarking. Petite histoire d'une technologie de gouvernement. " Revue d'histoire moderne et contemporaine, vol. 55-4 bis, 28-45.

Bruno, I. (2010). « La déroute du « benchmarking social ». » Revue française de socio-économie, vol. 1, 5, 41-61.

Bruno, I., \& Didier, E. (2013). Benchmarking. L'État sous pression statistique. Paris : Éditions La Découverte.

Références sur la notion d'institution

Calvez, M. (2006). « L'analyse culturelle de Mary Duglas : une contribution à la sociologie des institutions. "Sociologie, théories et recherches. Mis en ligne le 22 octobre 2006 et consulté le 25 juin 2012. URL : http : // sociologies.revues.org / 522.

Laforgue, D. (2009). "Pour une sociologie des institutions publiques contemporaines: Pluralité, hybridation et fragmentation du travail institutionnel. "Socio-logos, $N^{\circ} 4$. Mis en ligne le 23 septembre 2009 et consulté le 21 juin 2012. URL: http: // sociologos.revues.org / 2317.

Nantel, L. (2010). Conception du rapport individu/société : distinction fondamentale entre les concepts d'institution et d'organisation. Mémoire de Maîtrise de sociologie. Université du Québec à Montréal. 


\section{Résumé}

Une étude des publications anglaises, françaises et espagnoles sur les best practices a montré qu'elles ne constituent pas un genre, mais une multitude de types de pratiques dont les caractéristiques et la valeur varient grandement. En conséquence, le développement et la diffusion de best practices sont peu susceptibles d'aider les Comités internationaux de l'ICOM à améliorer profondément leurs productions. Un ensemble d'autres moyens semble plus approprié.

Mots clé : Best practices, excellence professionnelle

\section{Abstract}

Best Practices : The Treasure of Troy or the Treasure of Black Beard?

A review of the English, French and Spanish literature on best practices has shown that these are not one but many types of practices, varying greatly in their characteristics and values. So it does not seem that developing and disseminating best practices would really help the ICOM International Committees with strong professional objectives to seriously improve their production. A set of other means seem more relevant.

Key words: Best Practices, professional excellence

\section{Resumen}

¿Los Best Practices : tesoro de Troya o tesoro del pirata Barbarroja? Estudiando las publicaciones en español, francés y ingles sobre las buenas practicas, aquellas aparecieron no como un tipo, sino como numerosos tipos de practicas con varias características y niveles de excelencia. Entonces a los Comités internacionales del ICOM que poseen una fuerte orientación profesional, el desarrollo y la difusión de buenas practicas no ayudaría elevar el nivel de su producción. Otros medios parecen mas adecuados.

Palabras clave: buenas practicas, excelencia profesional 